\title{
ON CRITICAL CONDITION FOR A WEIGHTED INTEGRAL SYSTEM WITH NEGATIVE EXPONENTS
}

\section{LILI HUANG}

Abstract. This paper is concerned with the integral system

$$
\begin{cases}u(x)=\int_{R^{n}}|x|^{\alpha}|y|^{\beta}|x-y|^{s} v^{q}(y) d y, & u>0 \text { in } R^{n}, \\ v(x)=\int_{R^{n}}|x|^{\beta}|y|^{\alpha}|x-y|^{s} u^{p}(y) d y, & v>0 \text { in } R^{n},\end{cases}
$$

where $n \geqslant 1, \alpha, \beta, s>0$ and $p, q<0$. Such an integral system appears in the study of the conformal geometry and the weighted Hardy-Littlewood-Sobolev inequality. We obtain that

$$
\frac{1}{p+1}+\frac{1}{q+1}=-\frac{\alpha+\beta+s}{n},
$$

is a necessary condition for the existence of the $C^{1}$ positive entire solutions, which is also the necessary and sufficient condition for the invariant of the system and some energy functionals under the scaling transformation.

Mathematics subject classification (2010): 45E10, 45G15, 45M05, 45M20.

Keywords and phrases: Singular integral equation, critical condition, negative exponent.

\section{REFERENCES}

[1] G. Caristi, L. D’Ambrosio, E. Mitidieri, Representation formulae for solutions to some classes of higher order systems and related Liouville theorems, Milan J. Math., 76 (2008), 27-67.

[2] W. Chen, C. LI, Regularity of solutions for a system of integral equations, Commun. Pure Appl. Anal., 4 (2005), 1-8.

[3] W. Chen, C. Li, B. OU, Classification of solutions for an integral equation, Comm. Pure Appl. Math., 59 (2006), 330-343.

[4] C. Jin, C. Li, Symmetry of solutions to some systems of integral equations, Proc. Amer. Math. Soc., 134 (2006), 1661-1670.

[5] C. Jin, C. LI, Qualitative analysis of some systems of integral equations, Calc. Var. Partial Differential Equations, 26 (2006), 447-457.

[6] Y. LEI, On the integral systems with negative exponents, Discrete Contin. Dyn. Syst., 35 (2015), 1039-1057.

[7] Y. LEI, Critical conditions and finite energy solutions of several nonlinear elliptic PDEs in $R^{n}$, J. Differential Equations, 258 (2015), 4033-4061.

[8] Y. LEI, C. LI, C. MA, Asymptotic radial symmetry and growth estimates of positive solutions to weighted Hardy-Littlewood-Sobolev system, Calc. Var. Partial Differential Equations, 45 (2012), 4361.

[9] Y. LEI, Z. Lü, Axisymmetry of locally bounded solutions to an Euler-Lagrange system of the weighted Hardy-Littlewood-Sobolev inequality, Discrete Contin. Dyn. Syst., 33 (2013), 1987-2005.

[10] Y. LEI, C. MA, Asymptotic behavior for solutions of some integral equations, Commun. Pure Appl. Anal., 10 (2011), 193-207.

[11] C. LI, J. LIM, The singularity analysis of solutions to some integral equations, Commun. Pure Appl. Anal., 6 (2007), 453-464. 
[12] Y. LI, Remark on some conformally invariant integral equations: the method of moving spheres, J. Eur. Math. Soc., 6 (2004), 153-180.

[13] E. LIEB, Sharp constants in the Hardy-Littlewood-Sobolev and related inequalities, Ann. of Math., 118 (1983), 349-374.

[14] M. OnODERA, On the shape of solutions to an integral system related to the weighted HardyLittlewood-Sobolev inequality, J. Math. Anal. Appl., 389 (2012), 498-510.

[15] E. M. Stein, G. Weiss, Fractional integrals in $n$-dimensional Euclidean space, J. Math. Mech., 7 (1958), 503-514.

[16] X. XU, Uniqueness theorem for integral equations and its application, J. Funct. Anal., 247 (2007), 95-109.

[17] Y. ZHAO, Regularity and symmetry for solutions to a system of weighted integral equations, J. Math. Anal. Appl., 391 (2012), 209-222. 